\title{
MedienPädagogik
}

Themenheft Nr. 27: Tagungsband: Spannungsfelder und blinde Flecken. Medienpädagogik zwischen Emanzipationsanspruch und Diskursvermeidung. Herausgegeben von Sven Kommer, Thorsten Junge und Christiane Rust.

\section{Medienpädagogik als Kulturwissenschaft!? Überlegungen zu disziplinären Öffnungen und Anschlüssen}

Patrick Bettinger

\section{Zusammenfassung}

Der Beitrag diskutiert Möglichkeiten einer verstärkten Einbringung kulturwissenschaftlicher Perspektiven in die Medienpädagogik. Auf einem kulturwissenschaftlichen Programm im Anschluss an Reckwitz basierend, der Kultur als ein bedeutungsorientiertes und kontingenzgeprägtes Konstrukt begreift, wird gezeigt, wie diese Position durch ihre disziplinäre Querschnittslagerung ein Ensemble von alternativen Denk- und Analysemodellen anbietet, das medienpädagogische Fragestellungen bereichern kann. Wie sich mit Blick auf die jüngeren Diskussionen über disziplinäre Anschlüsse zwischen Erziehungswissenschaft und Kulturwissenschaft zeigt, sensibilisiert ein solcher Zugang für gegenwärtige gesellschaftliche Veränderungsprozesse und ermöglicht die Erschliessung heterogener und vielschichtiger Phänomene. Gerade aufgrund der zentralen Rolle der Medien in diesen Prozessen kann die Bedeutung einer kulturwissenschaftlichen Perspektivierung für die Medienpädagogik kaum überschätzt werden. Der Beitrag zeigt exemplarisch, wie kulturwissenschaftliche Anschlüsse der Medienpädagogik neue theoretische wie empirische Perspektiven eröffnen können, um komplexe Phänomene über ein praxeologisch-diskursanalytisch gerahmtes Forschungsprogramm zu erschliessen. Es wird dargelegt, wie sich hierdurch Lücken im medienpädagogischen Fachdiskurs schliessen lassen, die sich unter anderem im marginalisierenden Umgang mit Macht-Wissen-Komplexen, Subjektivität, Körperlichkeit, Materialität und Medialität feststellen lassen.

\section{Media education as cultural sciences!? - Thoughts on disciplinary openings and connections}

\begin{abstract}
This contribution discusses possibilities of fostering the implementation of a cultural sciences-perspective 1 into media education. Based on a program of cultural sciences referring to Reckwitz, who understands culture as a construct oriented towards meaning and contingency, the paper shows how this position offers an ensemble of alternative models for further discussion and analysis by its cross-sectional perspective

1 For the differentiation between cultural sciences and cultural studies see pp. 70
\end{abstract}


on disciplines that can enrich the media educational scope of questions. Such an approach sensitizes for current processes of societal change and enables the exploration of heterogenous and multilayered phenomena, as recent discussions show about disciplinary connections between educational science and cultural sciences. Just because of the central role of media in these processes a cultural sciences-perspective in media education can barely be overrated. This contribution exemplifies, how cultural sciences-based connections can establish new theoretical and empirical perspectives in media education in order to access complex phenomena by a research programme that is framed in terms of practices and discourses. The paper demonstrates how this approach can close gaps in the professional discourse of media education that can be located in the marginalising handling of complexes of power and knowledge, subjectivities, physicality, materiality and mediality.

\section{Einleitung}

Es scheint, als käme beinahe keine geistes- und sozialwissenschaftliche Disziplin ohne den Kulturbegriff aus. Dies gilt offensichtlich auch für die Erziehungswissenschaft, in der Schlagworte wie «kulturelle Bildungs oder ‘Lernkulturs ihren festen Platz haben und Kultur nicht zuletzt in Form einer 〈Kulturpädagogik〉 disziplinäre Verankerung erfahren hat. Spätestens seit dem «cultural turn` der 1970er Jahre (Reckwitz 2003, 287) etablierte sich ein Wandel in den Geistes- und Sozialwissenschaften, der zu einer radikalen Neuorientierung der analytischen Ausrichtung führte. Trotz des heterogenen Charakters der unter diesem Label begriffenen Ansätze (oder gerade deswegen) konnte diese Wende durch ihre disziplinäre Querlagerung bislang unerschlossene Forschungsbereiche zugänglich machen (vgl. Bachmann-Medick 2006, 7). Bei genauerer Betrachtung kann unter dem «cultural turn s eine Mehrzahl von nicht linear verlaufenden Wenden identifiziert werden, welche als Veränderungen von «Wahrnehmungseinstellungen, operativen Zugängen und Konzepten sowie von Analysekategorien» (ebd., 10) auftreten. Im Zentrum des Interesses stehen nun Fragen nach «Selbstauslegung und Inszenierung, Körperlichkeit und Handlungsmacht, aber auch die Politik sozialer und interkultureller Differenzen mit ihren Übersetzungs- und Aushandlungspraktiken» (ebd., 8). Diese Entwicklungen können als Antwort auf die Krise der traditionellen Geisteswissenschaften zum Ende des 21. Jahrhunderts verstanden werden, die sich zunehmend als Kulturwissenschaften verstehen um «einem wachsenden Bedürfnis theoretischer Neuorientierung» (List 2004, 6) zu begegnen. 
Es stellt sich vor diesem Hintergrund die Frage, inwiefern der «cultural turn` Auswirkungen auf die Medienpädagogik ${ }^{2}$ hatte oder gegenwärtig (noch) hat. Ob eine kulturwissenschaftliche Orientierung auch für diese (Teil-) Disziplin Möglichkeiten bietet, blinde Flecken zu beleuchten und wie eine solche Bereicherung aussehen könnte, versucht der vorliegende Beitrag zu umreissen. Insofern handelt es sich um eine programmatische Skizze, die zeigt, wo bislang Defizite in der medienpädagogischen Theorie und Empirie liegen und wie ein kulturwissenschaftliches Forschungsprogramm hierauf Antworten bieten kann. Hierzu wird zunächst der Kulturwissenschaftsbegriff präzisiert und 〈Kultur〉 als Brennpunkt eines spezifischen Forschungsprogramms entfaltet. Um der Frage nach Anschlussmöglichkeiten näher zu kommen, wirft der Beitrag anschliessend einen Blick auf disziplinäre Standortbestimmungen der Medienpädagogik und legt dar, inwiefern ein Selbstverständnis als erziehungswissenschaftliche Sub- oder Teildisziplin für das vorliegende Unterfangen fruchtbar ist. Dieser Schritt dient nicht nur der Erschliessung des diffusen Geflechts der umkämpften Zugehörigkeitsdiskurse der Disziplin, sondern soll deutlich machen, dass eine kulturwissenschaftliche Orientierung im hier dargelegten Verständnis in der Medienpädagogik bislang - wenn überhaupt - nur eine randständige Position hatte. Begreift man Medienpädagogik als Teildisziplin der Erziehungswissenschaft, so lassen sich verschiedene Aspekte finden, die für eine verstärkte Einbringung einer solchen kulturwissenschaftlichen Ausrichtung sprechen. Anknüpfend an bisherige Überlegungen stellt der Beitrag schliesslich exemplarisch Möglichkeiten einer solchen Verbindung vor und zeigt, wie eine am kulturwissenschaftlichen Forschungsprogramm ausgerichtete medienpädagogische Analyseeinstellung wenig beachtete Aspekte erschliessbar macht und so eine Bereicherung darstellt. Abschliessend werden die vorgestellten Überlegungen einer kritischen Prüfung unterzogen und es wird diskutiert, worin der Gewinn für die Medienpädagogik liegen kann, wenn sie sich verstärkt in Richtung der Kulturwissenschaften öffnet.

\section{Kulturwissenschaft(en) als quergelagertes Forschungsprogramm und diszip- linübergreifende Perspektive}

Bevor die zentralen Thesen des Beitrags entfaltet werden, soll zunächst eine grundlegende Klärung des dabei zugrunde gelegten Kulturverständnisses vorgenommen werden. Der Kulturbegriff erweist sich als äusserst polysem und ist in

2 Ist nachfolgend von ¿der〉 Medienpädagogik die Rede, so ist stets die deutschsprachige Medienpädagogik als wissenschaftliche (Sub-)Disziplin der Erziehungswissenschaft gemeint (näheres zur hier vorgenommenen disziplinären Verortung im Kapitel «Konturen disziplinärer Verortungen und Bezüge der Medienpädagogikı). Inwiefern sich die in diesem Beitrag umrissenen Kritikpunkte und Anregungen auch auf den internationalen Kontext der Medienpädagogik übertragen lassen, muss an dieser Stelle offen bleiben. 
seiner historischen Genese historisch von unterschiedlich in den Vordergrund tretenden, disziplinspezifischen Prägungen sowie Ab- und Eingrenzungen gekennzeichnet (Fuchs 2008, 7). Während die Beschäftigung mit Kultur gewissermassen das Kerngeschäft der Ethnologie darstellt (vgl. ebd., 105ff.) finden sich auch in der Soziologie, der Psychologie und der Erziehungswissenschaft in verschiedenster Form Bezüge zum Kulturellen. Erschwerend zu dieser begrifflichen Bedeutungsvielfalt kommt die alltagssprachliche Verwurzelung des Kulturbegriffs hinzu, der - ähnlich wie beim Begriff Bildung - suggeriert, es wäre scheinbar klar, wovon die Rede ist. Für eine wissenschaftliche Auseinandersetzung kann dies selbstverständlich nur ungenügend sein, weshalb an dieser Stelle eine Begriffsklärung vorgenommen werden soll. Um das Ausmass der Pluralität des diskursiven Feldes der Kulturwissenschaft(en) zu erahnen, hilft ein Blick in einschlägige Einführungsbände und Handbücher (vgl. z.B. List/Fiala 2004; Fuchs 2008; Jaeger/Liebsch 2011; Moebius/Quadflieg 2011a; Leggewie u. a. 2012). Die unter dem Dachbegriff Kulturwissenschaft firmierenden Ansätze weisen Bezüge zur Philosophie, Soziologie, Ethnologie, Literaturwissenschaft und viele andere mehr auf. Als Antwort auf diese Vielfalt finden sich entsprechend viele Systematisierungsversuche.

Reckwitz (2006a; 2011) folgend, wird in diesem Beitrag unter Kulturwissenschaften ein Forschungsprogramm verstanden, das bestimmte analytische Standpunkte einnimmt und Denkweisen sowie Fragerichtungen unter einer «Totalperspektive 〈Kultur〉» (Reckwitz 2011, 1) anregt. Unter dieser Prämisse lässt sich jeder Gegenstand aus dem Spektrum der Sozial- und Geisteswissenschaften als kulturelles Phänomen analysieren. Reckwitz (ebd., 2) definiert sein kulturwissenschaftliches Forschungsprogram wie folgt:

Das kulturwissenschaftliche Forschungsprogramm zielt darauf ab, die impliziten, in der Regel nicht bewussten symbolischen Ordnungen, kulturellen Codes und Sinnhorizonte zu explizieren, die in unterschiedlichsten menschlichen Praktiken - verschiedener Zeiten und Räume - zum Ausdruck kommen und diese ermöglichen.

Er unterscheidet einen normativen, einen totalitätsorientierten, einen differenzierungstheoretischen sowie einen bedeutungsorientierten Kulturbegriff und zeichnet deren Genese historisch nach (ebd.). Gerade das bedeutungsorientierte Kulturverständnis, welches auf der Grundlage der Annahme symbolisch vermittelter Ordnungsformen von Wirklichkeit als Fundament für die Entstehung und Veränderung historisch kontingenter Verhaltenskomplexe basiert, spiegelt für Reckwitz die aktuell bedeutendste Variante eines Kulturverständnisses wider und bildet den Hintergrund seines kulturwissenschaftlichen Forschungsprogramms (ebd., 7). Ein solcher, im weitesten Sinne als sozialkonstruktivistisch zu verstehender, Kulturbegriff speist sich aus einem Fundus von verschiedenen philosophischen und sozialtheoretischen Ansätzen mit phänomenologisch-hermeneutischer, 
strukturalistisch-semiotischer, pragmatistischer und sprachphilosophischer Prägung (ebd.). Eine zentrale Einsicht dieser Theorieströmungen stellt für Reckwitz die Annahme dar, dass alle menschlichen Lebensformen «nur im Verhältnis zu ihren spezifischen, kontingenten Sinnsystemen - gleichgültig, ob man diese Sinnsysteme nun als Zeichensysteme, Diskurse, Sinnhorizonte oder Sprachspiele konzeptualisieren mag» (ebd., 8) möglich sind und nur so als normal, rational, natürlich oder notwendig erscheinen. Für ein solches Kulturverständnis konstatieren Moebius und Quadflieg (2011b, 12), dass sich aus dieser Position heraus

[...] nicht nur bestimmte geistige und materielle Produktionen, sondern letztlich alle Wissensbestände und Praktiken als Ausdruck oder Erscheinungsweise einer spezifischen Kultur interpretieren [lassen]. Mit dem zeichen- und bedeutungstheoretisch gewendeten Kulturbegriff können entsprechend sowohl soziale Institutionen als auch mentale Dispositionen für kulturtheoretische Betrachtungen erschlossen werden; mit anderen Worten solche Gegenstandsbereiche, die vormals zum Kernbestand getrennter Disziplinen gehörten.

Indem Kultur systematisch als Kontingenzperspektive entfaltet wird, treten, wie Reckwitz $(2011,8)$ ausführt, Spannungen zu soziologischen, ethnologischen sowie geschichts- und literaturwissenschaftlichen Basisannahmen auf. Deren Tendenz zu einer «lnvisibilisierung von Kontingenz» (ebd.) gehe, so Reckwitz weiter, mit der Etablierung bestimmter wissenschafts- und gegenstandstheoretischer Rationalitäten einher, die in den angesprochenen Disziplinen starke Setzungen nach sich zogen (wie bspw. die Unterscheidung zwischen moderner und traditionaler Gesellschaft in der Soziologie) und aus Sicht eines bedeutungsorientierten Kulturverständnisses nicht mehr vollständig nachvollziehbar erscheinen. In seiner typisierenden Analyse differenziert Reckwitz unter dem Dach des bedeutungsorientierten Kulturbegriffs schliesslich zwei zentrale ‘Theoriefamilien〉, die er zunächst grob in ein praxistheoretisches und ein diskurstheoretisches Lager einteilt, die in der Tradition einer strukturalistisch-semiotischen bzw. einer phänomenologischhermeneutischen Position stehen (ebd., 14ff.).

Reckwitz bezeichnet sein kulturtheoretisches Analyseprogramm als ein komplexes und heterogenes Konglomerat von Ansätzen, «die den klassischen Sozialwissenschaften wie den klassischen Geisteswissenschaften einen Horizont neuartiger Fragestellungen eröffnen» (Reckwitz, 2006a, 705). In seiner auf Konvergenzen abzielenden Betrachtung grosser Theoriestränge gelingt es Reckwitz zu zeigen, wie die oft als Antagonisten verstandenen hermeneutisch-phänomenologischen und semiotisch-strukturalistischen Schulen in einem Forschungsprogramm vereint werden können, das in einem praxeologisch-diskurstheoretischen Fokus mündet. Damit einher geht für ihn die «Einführung und Verbreitung eines neuen erkenntnisleitenden Vokabulars, das neuartige analytische Perspektiven eröffnet» (ebd., 644). 
Eine in dieser Weise kulturwissenschaftlich gelagerte Forschungsperspektive ist somit stets ein interdisziplinäres Unterfangen (vgl. List 2004), welche die Aufmerksamkeit auf «Materialität, Medialität und Tätigkeitsformen des Kulturellen [richtet], um genauer zu erkennen, wie und in welchen Prozessen und kulturspezifischen Ausprägungen Geistiges und Kulturelles in einer jeweiligen Gesellschaft überhaupt produziert werden» (Bachmann-Medick 2006, 9). Die strikte Trennung, die u. a. zwischen mentalistischen und textualistischen Ansätzen oder zwischen Subjekt- und Objektwelt gezogen wurden, werden von einem solchen Kulturverständnis grundsätzlich in Frage gestellt. Indem das kulturwissenschaftliche Analyseprogramm die Verständnisse von Kultur als symbolische Strukturen sowie als Produkt subjektiver Interpretationen aufeinander bezieht, kann es als integrativer Ansatz gewertet werden, der ein weites Verständnis von Diskursen und sozialen Praktiken zu seinem analytischen Zentrum macht, die jeweils als «zwei aneinander gekoppelte Aggregatzustände der materiellen Existenz von kulturellen Wissensordnungen» (Reckwitz 2008, 202) verstanden werden. Unterteilt man das Fundament kulturwissenschaftlicher Arbeiten in die drei elementaren Bereiche 〈Symbol', ¿Gesellschaft) und 〈Individuum〉 (vgl. List 2004, 7), kann für gegenwärtige Kulturwissenschaften eine «intertheoretische Zugangsweise» (ebd.) ausgemacht werden, die disziplinäre Grenzen überwindet und den konstitutiven Zusammenhang dieser drei Bereiche betont. Eine solche «kulturwissenschaftliche Perspektivierung der Fragehorizonte» (Bachmann-Medick 2006, 19) kann dazu dienen, den analytischen Blick zu weiten und einem sich in dynamischem Wandel befindlichen Forschungsfeld neu zu begegnen.

Abgegrenzt werden muss das hier fokussierte Verständnis von Kulturwissenschaft von den Cultural Studies, die im Wesentlichen in den 1980er Jahren im angelsächsischen Raum durch Stuart Hall vorangetrieben wurden (vgl. Hepp 2008). Die Cultural Studies können als spezifischer Bereich der Kulturwissenschaften aufgefasst werden, der sich u. a. erstmalig der Populärkultur in wissenschaftlicher Absicht zugewendet hat (vgl. Winter 2011, 470). Cultural Studies-Analysen sind stets von einem kritischen Impetus geprägt und machen keinen Hehl aus ihrem Ehrgeiz, sich eindeutig im politischen Diskurs zu positionieren. Der Ansatz der Cultural Studies weist zwar einige Überschneidungspunkte mit dem hier zugrunde gelegten Verständnis eines kulturwissenschaftlichen Forschungsprogramms auf (bspw. die Betonung der Notwendigkeit eines interdisziplinären Zugangs zu Kultur), unterscheidet sich aber insofern davon, dass ihr Kernverständnis von einem «interventionistischen Charakter» (Winter 2004, 2) geprägt ist, und ein sehr spezifisches Kreislaufmodell von Kultur im Mittelpunkt steht (Hepp 2008, 144). Insofern sind die Cultural Studies trotz der Parallelen zu dem hier erläuterten kulturwissenschaftlichen Forschungsprogramm nicht mit diesem gleich zu setzen. 


\section{Konturen disziplinärer Verortungen und Bezüge der Medienpädagogik}

Bevor die Begründungsfigur einer «kulturwissenschaftlich informierten` Medienpädagogik entfaltet wird, soll zunächst ein Blick auf die Selbstverortung dieser (Teil-) Disziplin geworfen werden. Dass eine klare disziplinäre Zuordnung der Medienpädagogik schwierig bzw. nicht möglich ist, kann schon allein aufgrund der vielfältigen innerdisziplinären Strömungen, die historisch gewachsen sind und parallel existier(t)en, als grundlegend gelten. Dies zeigt ein Blick auf Arbeiten zur Geschichte der Medienpädagogik (bspw. in Sander, von Groß/Hugger 2008, 15ff.). Medienpädagogik weist vielfältige Bezugsdisziplinen auf (Hug 2002) und kann nicht umstandslos einer «Mutterdisziplin` zugeordnet werden, auch wenn allein aufgrund des Begriffs Bezüge zur Erziehungswissenschaft offensichtlich sind - und in Form einer Sektion in der Deutschen Gesellschaft für Erziehungswissenschaft (DGfE) auch institutionell etabliert. Neben der erziehungswissenschaftlichen Fundierung findet sich auch in der Kommunikationswissenschaft eine (als Fachgruppe in der Deutschen Gesellschaft für Publizistik und Kommunikationswissenschaft (DGPuK)) Verankerung der Medienpädagogik. Was Medienpädagogik ist oder sein soll, ist auch heute längst nicht geklärt, wie sich bspw. gegenwärtig an entsprechenden Veranstaltungen zeigt, die jeweils der Frage nach dem Selbstverständnis der Disziplin nachgehen ${ }^{3}$. Ob man so weit gehen muss, von einer traditionellen Zweiteilung in erziehungs- und kommunikationswissenschaftliche Medienpädagogik zu sprechen (vgl. Hartung/Schorb 2014, 8), sei an dieser Stelle dahingestellt. Einen anderen Vorschlag, Medienpädagogik intern auszudifferenzieren liefert Ruge (2014), der von drei Varianten von Medienpädagogik spricht, die jeweils Unterschiede in ihren Verhältnissen zum Medienbegriff, zu pädagogischen Kernbegriffen und ihrem wissenschaftlichen Habitus unterscheiden. Wie auch immer man zu den unterschiedlichen Verortungen und potenziell umkämpften und disparaten Positionierungen steht, so lässt sich zumindest als kleinster gemeinsamer Nenner festhalten, dass die Medienpädagogik sich «äußerst heterogen darstellt, sowohl in ihren theoretischen Ansätzen und methodischen Zugängen als auch in ihren Gegenständen» (Kerres et al. 2007, 8). Zentral für diesen Beitrag ist die Feststellung, dass Anschlüsse an eine kulturwissenschaftliche Perspektive, wie sie oben mit Reckwitz dargelegt wurde, bislang nur ansatzweise zu finden sind. Dies soll im Folgenden mit Blick auf medienpädagogische Grundlagenwerke, Einführungen und Periodi$k a$, von denen angenommen wird, dass sie das charakteristische theoretisch-empirische Spektrum der Disziplin abbilden, knapp veranschaulicht werden.

Hüther und Schorb (2010a) zählen (Medien-)Kultur(-analysen) nicht zu den medienpädagogischen Grundbegriffen und gehen auch in ihrer Definition von

3 Sowohl die Tagung der DGPuK-Fachgruppe Medienpädagogik sowie die Herbsttagung der DGfESektion Medienpädagogik widmeten sich 2016 der Frage nach dem Selbstverständnis der Medienpädagogik. 
Medienpädagogik nicht auf kulturwissenschaftliche Bezüge ein (2010b, 265ff.). Auch die Einführungen in die Medienpädagogik von Süss, Lampert und Wijnen (2013) oder Moser (2010) verweisen nicht explizit auf kulturwissenschaftliche Anschlüsse. Zwar taucht der Kulturbegriff in allen Bänden immer wieder in verschiedenen Zusammenhängen auf, von einer Orientierung der Medienpädagogik an neuere kulturwissenschaftliche Strömungen oder gar einem bedeutsamen Status des «cultural turn` für die (Sub-)Disziplin kann aber bislang offenbar nicht gesprochen werden. Auch ein Blick auf die Ausgaben des Jahrbuchs Medienpädagogik, das (neben der Online-Zeitschrift MedienPädagogik) als zentrales Publikationsorgan der Disziplin angesehen werden kann, revidiert diesen Eindruck nicht. In der Auseinandersetzung mit den Standortbestimmungen der Medienpädagogik (Sesink, Kerres und Moser 2007) finden sich lediglich bei Hug (2007), der sich dem «mediatic turn` widmet, ausgearbeitete Bezüge zum kulturwissenschaftlichen Feld. Ebenfalls kann im Jahrbuch Medienpädagogik 10 (vgl. Hartung et al. 2014), das sich «Methodologie und Methoden medienpädagogischer Forschung» verschrieben hat, kein Beitrag ausgemacht werden, welcher der Idee eines kulturwissenschaftlichen Forschungsprogramms im oben genannten Sinn entspricht und ausdrücklich eine praxeologisch-diskursanalytische Perspektive einnimmt. Stellenweise Erwähnung finden im medienpädagogischen Diskurs zumindest die Cultural Studies, so zum Beispiel in Form eines Beitrags von Hepp (2008) im Handbuch Medienpädagogik (Sander et al. 2008) oder einem Kapitel im Einführungsband von Moser (2010, 250ff.). Auch die bis 2009 bestehende Kasseler Medienpädagogik rund um Bachmair widmete sich den Cultural Studies (vgl. z. B. Bachmair 2007), die bspw. mit Prozessen der Mediensozialisation in Verbindung gebracht werden (vgl. Bachmair 2010a; 2010b). Insbesondere werden - aktuell im Rahmen der «London Mobile Learning Group - Bezüge zum kulturökologischen Ansatz hergestellt (vgl. Bachmair et al. 2014). Des Weiteren findet sich in Form eines Themenhefts der Zeitschrift MedienPädagogik von 2004 eine Auseinandersetzung mit der Frage nach dem Beitrag der Cultural Studies für die Medienpädagogik. Wie bereits oben erläutert, decken sich die Cultural Studies aber nicht mit dem kulturwissenschaftlichen Analyseprogramm, auf das dieser Beitrag rekurriert.

Ausgehend von einem Blick auf die disziplinären Verortungen der Medienpädagogik im Wissenschaftssystem soll gezeigt werden, dass aber durchaus grosses Potenzial besteht, eine stärkere Ausrichtung der Medienpädagogik an einem kulturwissenschaftlichen Forschungsprogramm zu forcieren. Begreift man Medienpädagogik als Teildisziplin der Erziehungswissenschaft (vgl. Spanhel 2007, 34f.) - die selbst wiederum stark mit unterschiedlichen Nachbardisziplinen verflochten ist und auf eine geisteswissenschaftliche Traditionslinie zurückblickt - so kann Medienpädagogik verstanden werden als Auseinandersetzung mit erziehungswissenschaftlich relevanten Fragestellungen (der Erziehung, des Lernens, der Bildung, 
der Sozialisation), die im Zusammenhang mit Medien betrachtet werden, wobei die Einbettung der untersuchten Phänomene in gesellschaftliche Kontexte berücksichtigt werden müssen ${ }^{4}$. Dieser, hier nur sehr grob skizzierte definitorische Ansatz, soll vorwiegend eines zeigen: Wenn Medienpädagogik im Sinne eines kulturwissenschaftlichen Analyseprogramms ausgelegt werden soll, muss die Frage nach ihren grundlegenden Bezügen geklärt werden. Ein erziehungswissenschaftlicher Kern scheint hierfür unter anderem deswegen sinnvoll, da innerhalb dieser Disziplin bereits seit einiger Zeit verstärkt kulturwissenschaftliche Anschlüsse diskutiert werden, wie im folgenden Kapitel veranschaulicht wird.

Insgesamt zeigen sich für die Medienpädagogik darüber hinaus einige Parallelen zum ebenfalls stark fragmentierten Feld der Kulturwissenschaften. In beiden Fällen handelt es sich quasi um 'Patchwork-Disziplinen), die sich aus unterschiedlichen Bezugsdisziplinen speisen bzw. diese in sich vereinen. So kann davon gesprochen werden, dass Interdisziplinarität und Multiperspektivität - mit allen positiven und negativen Konsequenzen - bereits im jeweiligen fachlichen Fundament angelegt sind. Wie die kulturwissenschaftliche Perspektive ist auch die Medienthematik in der Erziehungswissenschaft als eine quergelagerte anzusehen (Kerres et al. 2007, 8). Auch der von List (2004) für die Kulturwissenschaften konstatierte Bedarf nach theoretischer (und letztlich auch empirischer) Orientierung findet eine Entsprechung im Feld der Medienpädagogik, wie nicht zuletzt der auf die Klärung von Grundbegrifflichkeiten ausgerichtete Sammelband zu Medienbildung und Medienkompetenz von Moser, Grell und Niesyto (2011) zeigt. Festgehalten werden können für die Medienpädagogik immer wiederkehrende punktuelle Verweise auf die Cultural Studies, jedoch keine konsistenten Bemühungen, Anschlüsse an ein breiter begriffenes kulturwissenschaftliches Analyseprogramm zu suchen.

\section{Erziehungs- und kulturwissenschaftliche Konvergenzen}

Entgrenzungsbewegungungen und ein lebhafter Diskurs um Bezüge zu Nachbardisziplinen und programmatische Ausrichtungen können für die Erziehungswissenschaft seit jeher als typische Erscheinungen betrachtet werden (vgl. Baader 2013). Seit geraumer Zeit werden in der Erziehungswissenschaft - teilweise sehr offensiv - Stimmen laut, die eine stärkere Koppelung an die Kulturwissenschaften fordern. Sowohl Wimmer (2002) als auch Brumlik (2006) formulieren das Desiderat, Erziehungswissenschaft als Kulturwissenschaft (im Sinne der zuvor mit Reckwitz als «bedeutungsorientiert» attribuierten Variante) zu betreiben und begründen dies - wenn auch mit leicht abweichenden Argumentationsmustern - aus gesellschaftlichen Entwicklungen heraus, die eine Neuausrichtung eines kritischen

4 Siehe hierzu das Selbstverständnis der DGfE-Sektion Medienpädagogik: http://www.dgfe.de/sektionen-kommissionen/sektion-12-medienpaedagogik/ueber-die-sektion.html 
pädagogischen Selbstverständnisses erfordere. Aufgrund eines Gültigkeitsverlusts der Begriffe und damit der theoretischen Fundierung in der Erziehungswissenschaft, die unter anderem durch eine Pluralisierung von Diskursarten, einer unbestimmbaren Zunahme an Kontingenz sowie die medientechnischen Entwicklungen bedingt werden, sieht Wimmer (2002, 112f.) eine fundamentale Verunsicherung. Multiple Theoriebezüge in der Erziehungswissenschaft stellen für Wimmer in diesem Zusammenhang kein Manko dar, sondern werden von ihm als Option wahrgenommen, perspektivische Vielfalt in der Disziplin erkenntnisgewinnend umzusetzen. Für ihn ist die Erziehungswissenschaft einer «Erosion der Voraussetzungen einer kritischen Ortsbestimmung» (ebd., 114) ausgesetzt. Um diesem Missstand zu begegnen und die Autonomie der Pädagogik zu wahren, kann eine verstärkte Auseinandersetzung mit poststrukturalistischen Positionen gewinnbringend sein, deren Zusammengehörigkeit Wimmer letztlich unter dem Oberbegriff Kulturwissenschaft sieht (ebd., 115f.). Resümierend hält Wimmer (ebd., 117) fest:

Die kulturwissenschaftliche Wende in den Erziehungswissenschaften ist also als Wandel zur Multiperspektivität und Grenzverflüssigung zu verstehen, als Metaebene der Reflexion und als Form flexibler Verschaltung. So ist es möglich, einen Rahmen zu definieren, der es gestattet, Pluralität und Diversität nicht als Zerfallssymptom und Identitätsmangel zu bewerten, sondern als Reichtum, der Innovation, reflexive Relativierungen und Verbindungen nach außen ermöglicht.

Eine grundsätzliche, aber häufig nicht wahrgenommene Nähe der Erziehungswissenschaft zu den Kulturwissenschaften sieht Brumlik (2006, 62). Für ihn stellt das kulturwissenschaftliche Interesse an der Darstellung und dem Vollzug von Sinn den zentralen Bezugspunkt für die Pädagogik dar, deren Selbstverständnis für ihn insbesondere auf einer kritischen Auseinandersetzung mit ihren Gegenständen beruht (ebd., 63f.). Es scheint, folgt man den beiden Autoren, als würde in der Erziehungswissenschaft bereits mehr Kulturwissenschaft stecken, als man auf den ersten Blick annehmen könnte. Das disziplinäre Selbstverständnis sowie die Grundbegriffe der Pädagogik entsprechen in Teilen offenbar dem Anspruch der Kulturwissenschaften, «in einem praktischen Verhältnis zu den vielfältigen Formen kulturellen Lebens [zu stehen], die sie rekonstruieren» (Jaeger/Liebsch 2011, X). Ebenfalls kann die für die (Medien-)Pädagogik als Wissenschaft wesentliche Verbindung zur professionellen beruflichen Handlungspraxis, wie Wimmer $(2002,118)$ darlegt, über eine kulturwissenschaftliche Ausrichtung gewahrt werden:

Weil ihr kein spezifischer Objektbereich entspricht, kann Kulturwissenschaft als interdisziplinärer Rahmen auch einer Praxiswissenschaft fungieren, denn gerade weil sie an interpretativen und diskursanalytischen Verfahren orientiert ist und als soziale Praxis der Bedeutungsgenerierung angesehen werden kann, ist sie auch der Ort für Analysen der Wirksamkeit der Symbole, 
symbolischer Subsysteme und Handlungen auf die Subjekte, mithin auch pädagogischer Interaktionen und Institutionen.

Deutliche Verbindungslinien zwischen Erziehungs- und Kulturwissenschaft werden auch über Verweise auf den Stellenwert der Materialitäten für pädagogische Prozesse regelmässig herausgestellt. Für Rieger-Ladich (2009), der eine zunehmende Hybridisierung des Sozialen konstatiert, die den Bedarf nach neuen erziehungswissenschaftlichen Erkenntnismitteln bedingt, stellt gerade eine kulturwissenschaftliche Ausrichtung eine vielversprechende Möglichkeit dar, diesem Anspruch gerecht zu werden. Besonders in der vermehrt zu beobachtenden Zuwendung zu Artefakten sieht Rieger-Ladich (ebd., 120ff.) eine sich in der Erziehungswissenschaft bereits vollziehende Annäherung an kulturwissenschaftliches Denken. Auch bei Priem, König und Casale (2012) wird dargelegt, wie die Auseinandersetzung mit materiellen Dingen in Erziehung und Bildung von einer kulturwissenschaftlich inspirierten Forschungsperspektive profitieren kann. Die Herausgeberinnen machen sich für «eine erweiterte Forschungsperspektive, bei der Materialität als eine grundlegende und mehrdimensionale Kategorie einen zentralen Stellenwert für die Analyse von Erziehungs- und Bildungsprozessen einnimmt» (ebd., 9) stark. Inwiefern sich hier Kulturwissenschaften als Bündnispartner anbieten, macht insbesondere König (2012) in ihrem Beitrag deutlich. Sie erörtert den Facettenreichtum von analytischen Zugängen zum Materiellen und stellt heraus, wie bedeutungsorientierte Kulturanalysen (u. a. erziehungswissenschaftlicher Provenienz) nicht umhin kommen, sich in diesem Feld zu positionieren.

\section{Perspektiven einer kulturwissenschaftlich informierten Medienpädagogik}

Versteht man Lernen, Bildung, Erziehung und Sozialisation als kulturelle Phänomene in einer sich wandelnden und von heterogenen Diskurslandschaften durchzogenen Gesellschaft, so scheint die für die Erziehungswissenschaft bedenkenswerte Ausrichtung als Kulturwissenschaft (siehe vorheriges Kapitel) auch für eine sich im Kern als pädagogische (Sub-)Disziplin verstehende Medienpädagogik relevant zu sein. Nachfolgend soll daher beispielhaft expliziert werden, wie eine solche kulturwissenschaftliche Öffnung der Medienpädagogik aussehen könnte. Zur Veranschaulichung dieser These wurden Arbeiten ausgewählt, die eine deutliche Anschlussfähigkeit an medienpädagogische Fragestellungen und Themenfelder erkennen lassen oder diese bereits zumindest in Teilen schon aufgreifen. Die Darstellung kann selbstverständlich nicht als abschliessend angesehen werden, sondern soll lediglich anhand von ausgewählten Aspekten die zuvor erörterten Überlegungen konkreter werden lassen. Die herangezogenen thematischen Bezüge, in denen eine kulturwissenschaftliche Orientierung für die Medienpädagogik gewinnbringend erscheint, werden in der Auseinandersetzung mit dem 
Medien- bzw. Medialitätsbegriff, dem Subjektbegriff, dem analytischen Einbezug von Körperlichkeit und Materialität sowie Macht- und Herrschaftsphänomenen gesehen. Dabei soll nochmals betont werden, dass es eben nicht um die getrennte Betrachtung dieser Phänomene geht, sondern im Gegenteil um eine explizite Berücksichtigung von deren Verflechtungen.

Ein erster Ansatzpunkt kann in der aus medienpädagogischer Sicht unzureichenden Klärung des Medien- bzw. Medialitätsbegriffs bestehen. Wie Hug $(2007,13)$ feststellt, liegt «eine systematische Entfaltung von Medienbegriffen und deren Relevanz für medienpädagogische Fragestellungen, die breite Akzeptanz findet, [...] nicht vor». Zwar setzt der Band von Fromme und Sesink (2008) an genau dieser Problematik an, doch bildet dieser bis dato eher eine Ausnahme in der medienpädagogischen Theorielandschaft. Dabei erweist sich eine Verständigung darüber, auf welchen Medienbegriff man sich bezieht, gerade für die Medienpädagogik als zentrales Anliegen, da dieser richtungsweisende paradigmatische Impulse liefert. Indem ein kulturwissenschaftlicher Zugang gewählt wird, kann die Komplexität des Medienbegriffs, der symbolische, semiotische und materielle Dimensionen aufweist, handhabbar gemacht werden (vgl. Fiala 2004). Kulturwissenschaftliche Ansätze bieten darüber hinaus die Möglichkeit, sich verstärkt aus medienpädagogischer Sicht mit dem Subjektbegriff zu befassen. Grundsätzlich kann der Blick einer kulturwissenschaftlichen Subjektanalyse (vgl. Reckwitz 2012) medienpädagogische Fragestellungen bereichern, indem vermeintliche soziale Gegebenheiten, die bspw. im Zusammenhang mit Medien und Bildungsprozessen stehen, als soziohistorisch kontingente Erscheinungen erschlossen werden. Sozial-mediale Gesellschaftstransformationen und die daraus resultierenden Subjektformen (Reckwitz 2006b) können so als Hintergrundfolien für medienpädagogische Forschungskontexte herangezogen werden. Aus medienpädagogischer Sicht erscheint diesbezüglich der Band von Carstensen et al. (2014) sehr anregend. Indem bspw. über einen mehrperspektivischen Zugang, wie im Beitrag von Schachtner und Duller (2014), Zusammenhänge zwischen digitalen Praktiken von Kindern, Jugendlichen und Erwachsenen und Subjektivierungsformen untersucht werden, können Einblicke in die komplexen und teils ambivalenten «Hervorbringungsmodi〉 von Sozialität in digital geprägten Lebenswelten gewonnen werden.

Bezüglich der Körperlichkeit sind besonders neuere praxistheoretische Entwürfe die im Sinne eines bedeutungsorientierten Kulturbegriffs verstanden werden können - geeignet, um medienpädagogische Zugänge analytisch zu sensibilisieren (vgl. Schmidt 2012; Alkemeyer et al. 2013; Ferrin 2013). Begreift man Körperlichkeit als praxeologisch-diskursiv verfasst, können die gerade bei Jugendlichen relevanten medialen Inszenierungspraxen rekonstruiert und deren diskursive Kontextualisierung erschlossen werden. Indem soziale Praktiken in jüngeren poststrukturalistisch inspirierten Ansätzen nicht nur hinsichtlich ihrer Reproduktion betrachtet 
werden, sondern verstärkt in Bezug auf ihre Fragilität (vgl. Schäfer 2011; 2013), kann für den hier verfolgten Ansatz insbesondere die Frage nach der (In-)Stabilität medialer Praktiken im Zusammenhang mit formalen und informellen Lernund Bildungsprozessen hervorgehoben werden. Eng mit den neueren Ansätzen im Feld der Praxistheorien verbunden, ist auch die Frage nach dem Stellenwert der Materialität. So betonen bspw. Wieser (2006), Schäfer (2013) und Hillebrandt (2014) die praxistheoretische Nähe der Akteur-Netzwerk-Theorie (ANT) (z. B. Latour 2007), die Objekten einen zentralen Stellenwert in sozialen Gefügen einräumt und diesen den Status als wirkmächtige Grössen zuerkennt. Wie Lern-, Bildungsund Erziehungsprozesse über den Anschluss zur ANT die Materialitätsdimension konsequent berücksichtigen können zeigt Nohl (2011). Hier finden sich im Hinblick auf medienpädagogische Fragestellungen Ansatzpunkte, die spezifische Materialität(en) der Medien konzeptionell mitzudenken. Die Berücksichtigung materieller Artefakte als essentielle Bestandteile sozialer Praktiken in einem kulturtheoretischen Verständnis (vgl. Reckwitz 2010, 131ff.) kann gerade mit Blick auf die Verbreitung digitaler Medien in Form verschiedener mobiler Endgeräte von Smartphones bis hin zu Wearable-Technologies als bedeutsam für medienpädagogische Forschung betrachtet werden. Vielversprechend erscheinen auch die im Zuge des praxeologischen Revivals weiterentwickelten Forschungsmethoden, welche bspw. Fragen nach der Materialität der Praxis und ihrem Verhältnis zu gesellschaftlichen Wissensordnungen neu begegnen (vgl. Schäfer et al. 2015).

Als zweiter grosser Theoriestrang eines kulturwissenschaftlichen Forschungsprogramms kann neben den schwerpunktmässig praxistheoretisch ausgerichteten Zugängen das Areal der Diskursforschung ausgemacht werden. Zugänge dieser Ausrichtung sind in der Medienpädagogik so gut wie nicht vertreten. Zwar finden sich bereits vereinzelt Arbeiten, die sich mit Macht- und Herrschaftsphänomenen sowie damit zusammenhängenden Fragen (z. B. nach hegemonialen Subjektivierungsmustern oder widerständigen Subjektivationen) aus medienpädagogischer Perspektive befassen (z. B. Hipfl 2004; Fromme/Schäffer 2007). Erstaunlicher Weise spielen jedoch diskurs-, dispositiv- oder gouvernementalitätsanalytische Zugänge, die sich ausdrücklich medienpädagogisch verstehen, bis auf wenige Ausnahmen (z. B. Hoffarth 2013; 2015; Münte-Goussar 2011; 2015) keine Rolle in der Medienpädagogik. Dabei sind gerade die im Anschluss an Foucault orientierten Analysen durch ihren Blick auf das Zusammenspiel von Wissen und Macht erziehungswissenschaftlich hoch relevant (vgl. Schäffer 2007, 163). Im wachsenden Fundus der in jüngerer Vergangenheit - hauptsächlich mit soziologischem Schwerpunkt - entstandenen Publikationen, die sich mit diskursiven Phänomenen und Medien befassen (z. B. Dreesen et al. 2012; Fraas et al. 2013), finden sich bis auf den Sammelband von Othmer und Weich (2015) so gut wie keine Beiträge, welche sich aus medienpädagogischer Perspektive dezidiert einer diskursanalytischen Herangehensweise 
widmen (vgl. hierzu auch den Beitrag von Dander in diesem Themenheft). Selbst in dem mit «Diskursive und produktive Praktiken in der digitalen Kultur» betitelten Jahrbuch Medienpädagogik 11 (Kammerl et al. 2014) lässt sich kein Beitrag ausmachen, der einer diskursanalytischen Position zugeordnet werden kann. Erstaunlich scheint dieser Befund nicht nur aufgrund dem aus (medien)pädagogischer Sicht höchst relevant erscheinenden Anliegen der Diskursforschung, kollektive Wissensordnungen zu rekonstruieren und deren (sozial-medialen) Konstruktionslogiken als Basis der Wirklichkeitsaushandlungen offenzulegen (vgl. Keller 2011, 13ff.). Diskursanalytisch gewonnene Einsichten zeigen Muster der Positionierung innerhalb bestimmter Wissensordnungen und können als Fundament der Reflexion medienpädagogischen Handelns herangezogen werden. Die möglichen Erkenntnispotenziale und Einsatzmöglichkeiten einer erziehungswissenschaftlich ausgerichteten Diskursforschung untermauern Wrana et al. (2014) sowie Fegter et al. (2015). Dass eine solche Herangehensweise selbst für didaktische Fragestellungen anschlussfähig ist, zeigen Maier et al. (2012) in ihrer praxeologisch-diskursanalytisch ausgerichteten Arbeit zur Rekonstruktion von Lesarten im Professionalisierungsprozess. Es kann festgehalten werden, dass nicht nur die Fragen nach dem Medien- und Subjektbegriff über kulturwissenschaftlich inspirierte Ansätze fruchtbar gemacht, sondern auch die Kategorien Körperlichkeit und Materialität insbesondere über praxeologische Zugänge rehabilitiert werden können (Reckwitz 2011, 18). Die diskursanalytische Perspektive ermöglicht darüber hinaus besonders die Erschliessung von überindividuellen Macht-Wissen-Komplexen und deren subjektivierende Wirkungen. Wie mit Verweis auf das kulturwissenschaftliche Forschungsprogramm gezeigt wurde, sind die derart gelagerten Ansätze nicht als Gegensätze zu verstehen, sondern können als komplementäre Zugänge verstanden werden, die gerade durch ihre spezifischen Sichtweisen auf symbolische Ordnungen und deren (Re-)Produktion als Grundpfeiler des Sozialen der Medienpädagogik die Möglichkeit bieten, ihrem Gegenstandbereich neu zu begegnen. So kann der Blick für Interdependenzen zwischen den einzelnen Facetten der Phänomene geschärft werden, die sich unter anderem zwischen Materialität und Medialität oder dem Verhältnis von Subjektivierung und Körperlichkeit zeigen.

\section{Fazit}

Medienpädagogische Anschlüsse an eine kulturwissenschaftliche Analytik fragen nach Zusammenhängen sozialer Phänomene (vgl. Reckwitz 2006a, 722). Dabei kann dieser Position tendenziell ein holistischer Anspruch unterstellt werden, indem verschiedene Seiten der untersuchten Phänomene in ihrem Zusammenspiel betrachtet werden und so bewusst nicht nur seine Seite der Medailles die Forschungspraxis anleitet. Wie gezeigt wurde, finden sich im Bereich der Medienpädagogik 
bislang lediglich vereinzelt Arbeiten, die einem solchen Forschungsprogramm folgen. Eine kulturwissenschaftlich ausgerichtete Medienpädagogik kann letztlich als mehrperspektivisch verstanden werden, indem die Verschränkung sozialer Phänomene auf unterschiedlichen Ebenen bedacht wird. Eine Stärke offenbart sich in der Möglichkeit eines solchen Zugangs, auf die Herausforderungen gegenwärtiger gesellschaftlicher Veränderungen reagieren zu können, ob nun - global gefasst - als modernisierungsbedingte Pluralisierung von Entscheidungsoptionen durch zunehmende Entgrenzung (Beck/Lau 2004) oder spezifischer als medienkultureller Wandel (Hepp 2013). Ein solches Vorgehen rückt selbstverständlich Fragen nach (Un-)Vereinbarkeiten der damit adressierten epistemologischen Fundamente in den Mittelpunkt, wie beispielhaft an dem Verhältnis von Praktiken und Diskursen (vgl. z. B. Reckwitz 2008; Wrana/Langer 2007) deutlich wird. Um nicht der Gefahr eines (anything goes) und wahllosen Eklektizismen zu erliegen, bedarf es fundierten begrifflichen Erörterungen, denn «Begriffe stehen für Theorien» (Spanhel 2011, 97) und diese wiederum sind die Grundpfeiler wissenschaftlichen Schaffens - auch in der Empirie (vgl. Kalthoff et al. 2008). Anknüpfungsversuche an kulturwissenschaftliche Positionen können nicht nur dazu beitragen, empirische Analysen methodologisch und methodisch anzuleiten, sondern bieten zudem die Chance, medienpädagogische Theoriebildung voranzutreiben. Dies scheint insofern evident, da der programmatische Gestus praxeologischer und diskurstheoretischer Zugänge nicht dazu nötigt, medienpädagogische Theorien unter bereits bestehende Ansätze zu subsumieren, sondern im Gegenteil kann deren Offenheit als Angebot aufgefasst werden, gerade nicht einfach einem «Baukastenprinzip〉 in der Theoriebildung zu folgen, sondern Ansätze weiterzuentwickeln und disziplinspezifisch zu formen. Somit lässt sich auch dem von Hüther und Schorb (2010b, 273f.) konstatierten Bedeutungswandel der Medien begegnen, welcher als weitreichende Vereinnahmung aller Lebensbereiche durch Medien komplexe Denk- und Handlungsansätze erforderlich machen und der Medienpädagogik abverlangt, dass sie «mehr als bisher die Zusammenhänge zwischen den technologischen, pädagogischen, politischen und ökonomischen Implikationen der Medien» (ebd.) berücksichtigt.

Darüber hinaus stellt die kulturwissenschaftliche Perspektive auch eine Möglichkeit für die Medienpädagogik dar, sich selbst reflexiv in den Blick zu nehmen und die Fragen nach der Selbstverortung im Wissenschaftssystem zu beantworten. Nimmt man an, dass grundbegriffliche Positionierungen und Annahmen die Basis für disziplinäre Strukturen und Forschungspraxis darstellen (Reckwitz 2011, 2f.), so wäre eine genealogische Aufarbeitung genau dieser Prozesse für die Medienpädagogik ein wichtiger Schritt auf dem Weg zu disziplinärer Selbsterkenntnis (vgl. Bettinger im Druck). Damit die ohnehin schon durch multiple Bezugsdisziplinen und entsprechender perspektivischer Vielfalt geprägte Medienpädagogik im Zuge solcher Anschlüsse nicht noch mehr zersplittert und im umkämpften Feld der 
Wissenschaft nicht ihre Eigenständigkeit verliert, bedarf es grundsätzlichen konsolidierenden Überlegungen. Ein Aspekt kann und sollte hier die Weiterführung der Klärung von Grundbegriffen sein, die letztlich ganz entscheidend dafür sind, wie sich eine Disziplin selbst versteht und verortet - sowohl in den Feldern von Wissenschaft und Praxis wie auch im öffentlichen und politischen Diskurs.

Es stellt sich abschliessend die Frage, ob eine Öffnung der Medienpädagogik für die Kulturwissenschaften möglich ist, ohne ein zunehmendes Aufweichen der disziplinären Grenzen in Kauf nehmen zu müssen, das die ohnehin schon schwierige Selbstverortung noch zunehmend erschwert. Um derlei Anschlüsse auf lange Sicht möglichst fruchtbar zu gestalten, sollte daher - so die hier verfolgte These - ein klares Bekenntnis zum erziehungswissenschaftlichen Kern der Medienpädagogik die Grundlage bilden. Hiervon ausgehend könnte eine solche Öffnung als «disziplinäre Evolution` gestaltet und Entgrenzungsprozesse nicht der Beliebigkeit überlassen werden. Zu überlegen wäre, ob die medienpädagogischen Teilbereiche, welche als unterschiedliche Zugänge der pädagogischen Beschäftigung mit Medien verstanden werden können (vgl. Ruge 2014), stärker integriert und aufeinander bezogen werden müssten, um eine Zersplitterung zu vermeiden. Trotz der angesprochenen Hürden kann dies ein Weg sein, dem medienpädagogischen Erkenntnisgewinn neue Inspiration zu verschaffen. Eine kulturwissenschaftlich inspirierte Medienpädagogik bietet die Möglichkeit, blinde Flecken der Disziplin zu erhellen und ihr darüber hinaus im umkämpften Feld der Wissenschaft durch Stärkung ihrer Legitimationsgrundlagen neues Selbstbewusstsein zu verleihen.

\section{Literatur}

Alkemeyer, Thomas, Gunilla Budde, und Dagmar Freist, Hrsg. 2013. Selbst-Bildungen. Soziale und kulturelle Praktiken der Subjektivierung. Bielefeld: transcript.

Baader, Meike Sophia. 2013. «Erziehungswissenschaft zwischen disziplinären Grenzen, Grenzüberschreitungen und Entgrenzungen.» In Erziehungswissenschaftliche Grenzgänge. Markierungen und Vermessungen. Beiträge zum 23. Kongress der Deutschen Gesellschaft für Erziehungswissenschaft, hrsg. v. Hans-Rüdiger Müller, Sabine Bohne, und Werner Thole, 61-80. Schriften der Deutschen Gesellschaft für Erziehungswissenschaft (DGfE). Opladen: Budrich.

Bachmair, Ben. 2007. «Mediensozialisation - die Frage nach Sozialisationsmustern im Kontext dominanter Medienformen.» In Jahrbuch Medienpädagogik 6. Medienpädagogik - Standortbestimmung einer erziehungswissenschaftlichen Disziplin, hrsg. v. Werner Sesink, Michael Kerres, und Heinz Moser, 118-143. Wiesbaden: VS Verlag für Sozialwissenschaften.

Bachmair, Ben. Hrsg. 2010a. Medienbildung in neuen Kulturräumen. Die deutschsprachige und britische Diskussion. Wiesbaden: VS Verlag für Sozialwissenschaften. 
Bachmair, Ben. 2010b. «Mediensozialisation: Entwicklung von Subjektivität in medialen und kulturellen Figurationen.» In Mediensozialisationstheorien. Modelle und Ansätze in der Diskussion, hrsg. v. Dagmar Hoffmann und Lothar Mikos, 67-91. 2. Aufl. Wiesbaden: VS Verlag für Sozialwissenschaften.

Bachmair, Ben, John Cook, und Norbert Pachler. 2014. «Mobile Medien als Kulturressourcen für Lernen, ein kulturökologischer Beitrag zur Medienbildung.» In Perspektiven der Medienbildung, hrsg. v. Winfried Marotzki und Norbert Meder, 209-233. Wiesbaden: Springer.

Bachmann-Medick, Doris. 2006. Cultural Turns. Neuorientierungen in den Kulturwissenschaften. Reinbek bei Hamburg: Rowohlt.

Bettinger, Patrick. Im Druck. «Medienpädagogik: ein Kampfsport? Diskursive Positionierungen als Antworten auf die Frage nach dem disziplinären Selbstverständnis». In Medienpädagogik - Was ist das?, hrsg. v. Christine Trültzsch-Wijnen und Anja Hartung-Griemberg. Baden-Baden: NOMOS.

Brumlik, Micha. 2006. " "Kultur" ist das Thema. Pädagogik als kritische Kulturwissenschaft». Zeitschrift für Pädagogik 52, Nr. 1: 60-68.

Dreesen, Philipp, Łukasz Kumięga, und Constanze Spieß, Hrsg. 2012. Mediendiskursanalyse. Diskurse - Dispositive - Medien - Macht. Wiesbaden: VS.

Beck, Ulrich, und Christoph Lau, Hrsg. 2004. Entgrenzung und Entscheidung. Was ist neu an der Theorie reflexiver Modernisierung? Edition Zweite Moderne. Frankfurt am Main: Suhrkamp.

Carstensen, Tanja, Christina Schachtner, Heidi Schelhowe, und Raphael Beer, Hrsg. 2014. Digitale Subjekte. Praktiken der Subjektivierung im Medienumbruch der Gegenwart. Bielefeld: transcript.

Fegter, Susann, Fabian Kessl, Antje Langer, Marion Ott, Daniel Rothe, und Daniel Wrana, Hrsg. 2015. Erziehungswissenschaftliche Diskursforschung. Empirische Analysen zu Bildungs- und Erziehungsverhältnissen. Interdisziplinäre Diskursforschung. Wiesbaden: Springer.

Ferrin, Nino. 2013. Selbstkultur und mediale Körper. Zur Pädagogik und Anthropologie neuer Medienpraxen. Körperkulturen. Bielefeld: transcript.

Fiala, Erwin. 2004. "Medien, Zeichen und Kultur. Medientheoretische und semiologische Grundlagen der Kulturwissenschaft.» In Grundlagen der Kulturwissenschaften. Interdisziplinäre Kulturstudien, hrsg. v. Elisabeth List und Erwin Fiala, 99-118. Tübingen und Basel: A. Francke Verlag.

Fromme, Johannes, und Burkhard Schäffer, Hrsg. 2007. Medien - Macht - Gesellschaft. Wiesbaden: VS Verlag für Sozialwissenschaften.

Fromme, Johannes, und Werner Sesink, Hrsg. 2008. Pädagogische Medientheorie. Wiesbaden: VS Verlag für Sozialwissenschaften.

Fuchs, Max. 2008. Kultur Macht Sinn. Wiesbaden: VS Verlag für Sozialwissenschaften.

Hartung, Anja, und Bernd Schorb. 2014. «Methodologie und Methoden medienpädagogischer Forschung. Hintergrund, Idee und Anlage des Buches.» In Jahrbuch Medienpädagogik 10. Methodologie und Methoden medienpädagogischer Forschung, hrsg. v. Anja Hartung, Bernd Schorb, Horst Niesyto, Heinz Moser, und Petra Grell, 7-24. Wiesbaden: VS Verlag für Sozialwissenschaften.

Hartung, Anja, Bernd Schorb, Horst Niesyto, Heinz Moser, und Petra Grell, Hrsg. 2014. Jahrbuch Medienpädagogik 10. Methodologie und Methoden medienpädagogischer Forschung. Wiesbaden: VS Verlag für Sozialwissenschaften. 
Hepp, Andreas. 2008. "Cultural Studies als Ansatz der Medien- und Kulturforschung.»In Handbuch Medienpädagogik, hrsg. v. Uwe Sander, Friederike von Gross, und Kai-Uwe Hugger, 142-148. Wiesbaden: VS Verlag für Sozialwissenschaften.

Hepp, Andreas. 2013. Medienkultur. Die Kultur mediatisierter Welten. 2. Aufl. Wiesbaden: VS Verlag für Sozialwissenschaften.

Hipfl, Brigitte. 2004. «Medien - Macht - Pädagogik. Konturen einer Cultural-Studies-basierten Medienpädagogik, illustriert an Reality-TV-Sendungen». MedienPädagogik. Zeitschrift für Theorie und Praxis der Medienbildung Themenheft 8: 1-22. http://www. medienpaed.com/article/view/49.

Hoffarth, Britta. 2013. "Schmutzige Witze. Erkundung eines Bildungsereignisses im Geschlechter-Dispositiv.» In Verortungen des Dispositiv-Begriffs, hrsg. v. Joannah Caborn Wengler, Britta Hoffarth, und Łukasz Kumięga, 91-110. Wiesbaden: Springer.

Hoffarth, Britta. 2015. «Ungeheure Weiber. Monster, Bildung, Dispositiv.» In Medien - Bildung - Dispositive, hrsg. v. Julius Othmer und Andreas Weich, 203-220. Wiesbaden: Springer VS.

Hug, Theo. 2002. «Medienpädagogik - Begriffe, Konzeptionen, Perspektiven.» In Einführung in die Medienwissenschaft, hrsg. v. Gebhard Rusch, 189-207. Opladen: Westdeutscher Verlag.

Hug, Theo. 2007. «Medienpädagogik unter den Auspizien des mediatic turn - eine explorative Skizze in programmatischer Absicht.» In Jahrbuch Medienpädagogik 6. Medienpädagogik - Standortbestimmung einer erziehungswissenschaftlichen Disziplin, hrsg. v. Werner Sesink, Michael Kerres, und Heinz Moser, 10-32. Wiesbaden: VS Verlag für Sozialwissenschaften.

Hüther, Jürgen, und Bernd Schorb, Hrsg. 2010a. Grundbegriffe Medienpädagogik. 5. Aufl. München: kopaed.

Hüther, Jürgen, und Bernd Schorb. 2010b. «Medienpädagogik.» In Grundbegriffe Medienpädagogik, hrsg. v. Jürgen Hüther und Bernd Schorb, 265-276. 5. Aufl. München: kopaed.

Jaeger, Friedrich, und Burkhard Liebsch. 2011. «Einführung.»In Handbuch der Kulturwissenschaften, Band 1, hrsg. v. Friedrich Jaeger und Burkhard Liebsch, Grundlagen und Schlüsselbegriffe: IX-XIII. Stuttgart \& Weimar: Metzler.

Kalthoff, Herbert, Stefan Hirschauer, und Gesa Lindemann, Hrsg. 2008. Theoretische Empirie. Zur Relevanz qualitativer Forschung. Frankfurt am Main: Suhrkamp.

Kammerl, Rudolf, Alexander Unger, Petra Grell, und Theo Hug, Hrsg. 2014. Jahrbuch Medienpädagogik 11. Diskursive und produktive Praktiken in der digitalen Kultur. Wiesbaden: VS Verlag für Sozialwissenschaften.

Keller, Reiner. 2011. Diskursforschung. Eine Einführung für Sozialwissenschaftlerlnnen. 4. Aufl. Wiesbaden: VS Verlag für Sozialwissenschaften.

Kerres, Michael, Heinz Moser und Werner Sesink. 2007. «Medienpädagogik - Standortbestimmung einer erziehungswissenschaftlichen Disziplin - Editorial -.» In Jahrbuch Medienpädagogik 6. Medienpädagogik - Standortbestimmung einer erziehungswissenschaftlichen Disziplin, hrsg. v. Werner Sesink, Michael Kerres, und Heinz Moser, 7-8. Wiesbaden: VS Verlag für Sozialwissenschaften.

König, Gudrun M. 2012. «Das Veto der Dinge. Zur Analyse materieller Kultur.» In Die Materialität der Erziehung. Kulturelle und soziale Aspekte pädagogischer Objekte, hrsg. v. Karin Priem, Gudrun M. König, und Rita Casale, 14-31. Zeitschrift für Pädagogik, Beiheft 58. Weinheim und Basel: Beltz. 
Latour, Bruno. 2007. Eine neue Soziologie für eine neue Gesellschaft. Einführung in die Akteur-Netzwerk-Theorie. Frankfurt am Main: Suhrkamp.

Leggewie, Claus, Dariuš Zifonun, Anne Lang, Marcel Siepmann, und Johanna Hoppen, Hrsg. 2012. Schlüsselwerke der Kulturwissenschaften. Edition Kulturwissenschaft Bd. 7. Bielefeld: transcript.

List, Elisabeth. 2004. «Einleitung. Intedisziplinäre Kulturforschung auf der Suche nach theoretischer Orientierung.» In Grundlagen der Kulturwissenschaften. Interdisziplinäre Kulturstudien, hrsg. v. Elisabeth List und Erwin Fiala, 3-12. Tübingen und Basel: A. Francke Verlag.

List, Elisabeth, und Erwin Fiala, Hrsg. 2004. Grundlagen der Kulturwissenschaften. Interdisziplinäre Kulturstudien. Tübingen und Basel: A. Francke Verlag.

Maier Reinhard, Christiane, Barbara Ryter Krebs, und Daniel Wrana. 2012. «Lesarten im Professionalisierungsprozess. Eine empirische Analyse der Verstehensprozesse in Lernberatungsgesprächen.» In Professionalisierung in Lernberatungsgesprächen. Theoretische Grundlegungen und empirische Untersuchungen, hrsg. v. Daniel Wrana und Christiane Maier Reinhard, 69-160. Opladen: Budrich.

Moebius, Stephan, und Dirk Quadflieg, Hrsg. 2011a. Kultur. Theorien der Gegenwart. 2. Aufl. Wiesbaden: VS Verlag für Sozialwissenschaften.

Moebius, Stephan, und Dirk Quadflieg. 2011b. «Kulturtheorien der Gegenwart - Heterotopien der Theorie.» In Kultur. Theorien der Gegenwart, hrsg. v. Stephan Moebius und Dirk Quadflieg, 11-18. 2. Aufl. Wiesbaden: VS Verlag für Sozialwissenschaften.

Moser, Heinz. 2010. Einführung in die Medienpädagogik. Aufwachsen im Medienzeitalter. 5. Aufl. Wiesbaden: VS Verlag für Sozialwissenschaften.

Moser, Heinz, Petra Grell, und Horst Niesyto, Hrsg. 2011. Medienbildung und Medienkompetenz. Beiträge zu Schlüsselbegriffen der Medienpädagogik. München: kopaed. doi:10.21240/mpaed/20.X.

Münte-Goussar, Stephan. 2011. «Ambivalente Selbst-Techniken: Portfolio, Ökonomisierung, Selbstbestimmung.» In Kontrolle und Selbstkontrolle. Zur Ambivalenz von E-Portfolios in Bildungsprozessen, hrsg. v. Torsten Meyer, Kerstin Mayrberger, Stephan Münte-Goussar, und Christina Schwalbe, 225-250. Wiesbaden: VS Verlag für Sozialwissenschaften.

Münte-Goussar, Stephan. 2015. «Dispositiv - Technologien des Selbst - Portfolio.» In Medien - Bildung - Dispositive, hrsg. v. Julius Othmer und Andreas Weich, 109-127. Wiesbaden: VS Verlag für Sozialwissenschaften.

Othmer, Julius und Andreas Weich, Hrsg. 2015. Medien - Bildung - Dispositive. Beiträge zu einer interdisziplinären Medienbildungsforschung. Wiesbaden: VS Verlag für Sozialwissenschaften.

Priem, Karin, Gudrun M. König, und Rita Casale, Hrsg. 2012. «Die Materialität der Erziehung. Kulturelle und soziale Aspekte pädagogischer Objekte.» Zeitschrift für Pädagogik, Beiheft 58. Weinheim und Basel: Beltz.

Reckwitz, Andreas. 2003. "Grundelemente einer Theorie sozialer Praktiken. Eine sozialtheoretische Perspektive.» Zeitschrift für Soziologie 32, Nr. 4: 282-301.

Reckwitz, Andreas. 2006a. Die Transformation der Kulturtheorien. Zur Entwicklung eines Theorieprogramms. Weilerswist: Velbrück Wissenschaft.

Reckwitz, Andreas. 2006b. "Die historische Transformation der Medien und die Geschichte des Subjekts.» In Medien der Gesellschaft - Gesellschaft der Medien, hrsg. v. Andreas Ziemann, 89-107. Konstanz: UVK. 
Reckwitz, Andreas. 2008. «Praktiken und Diskurse. Eine sozialtheoretische und methodologische Relation.» In Theoretische Empirie. Zur Relevanz qualitativer Forschung, hrsg. v. Herbert Kalthoff, Stefan Hirschauer, und Gesa Lindemann, 188-209. Frankfurt am Main: Suhrkamp.

Reckwitz, Andreas. 2010. Unscharfe Grenzen. Perspektiven der Kultursoziologie. 2. Aufl. Bielefeld: transcript.

Reckwitz, Andreas. 2011. «Die Kontingenzperspektive der Kultur. Kulturbegriffe, Kulturtheorien und das kulturwissenschaftliche Forschungsprogramm». In Handbuch der Kulturwissenschaften, Band 3, hrsg. v. Friedrich Jaeger und Jörn Rüsen: Themen und Tendenzen: 1-20. 2. Aufl. Stuttgart \& Weimar: Metzler.

Reckwitz, Andreas. 2012. Subjekt. 3. Aufl. Bielefeld: transcript.

Rieger-Ladich, Markus. 2009. «Menschen und Dinge, Akteure und Aktanten. Überlegungen zur Neubestimmung des Sozialen.» In Das Soziale in der Pädagogik. Zürcher Festgabe für Reinhard Fatke, hrsg. v. Bettina Grubenmann und Reinhard Fatke, 114-130. Bad Heilbrunn: Klinkhardt.

Ruge, Wolfgang B. 2014. «Pädagogik * Medien - Eine Suchanfrage.» In Perspektiven der Medienbildung, hrsg. v. Winfried Marotzki und Norbert Meder, 187-207. Wiesbaden: VS Verlag für Sozialwissenschaften.

Sander, Uwe, Friederike von Gross, und Kai-Uwe Hugger, Hrsg. 2008. Handbuch Medienpädagogik. Wiesbaden: VS Verlag für Sozialwissenschaften.

Schachtner, Christina, und Nicole Duller. 2014. "Kommunikationsort Internet. Digitale Praktiken und Subjektwerdung.» In Digitale Subjekte. Praktiken der Subjektivierung im Medienumbruch der Gegenwart, hrsg. v. Tanja Carstensen, Christina Schachtner, Heidi Schelhowe, und Raphael Beer, 81-154. Bielefeld: transcript.

Schäfer, Hilmar. 2011. "Bourdieu gegen den Strich lesen. Eine poststrukturalistische Perspektive.» In: Pierre Bourdieu und die Kulturwissenschaften. Zur Aktualität eines undisziplinierten Denkens, hrsg. v. Daniel Suber, Hilmar Schäfer, und Sophia Prinz, 63-85. Konstanz: UVK.

Schäfer, Hilmar. 2013. Die Instabilität der Praxis. Reproduktion und Transformation des Sozialen in der Praxistheorie. Weilerswist: Velbrück Wissenschaft.

Schäfer, Franka, Anna Daniel, und Frank Hillebrandt, Hrsg. 2015. Methoden einer Soziologie der Praxis. Bielefeld: transcript.

Spanhel, Dieter. 2007. «Zur Standortbestimmung der Medienpädagogik aus anthropologischer und bildungswissenschaftlicher Sicht.» In Jahrbuch Medienpädagogik 6. Medienpädagogik - Standortbestimmung einer erziehungswissenschaftlichen Disziplin, hrsg. v. Werner Sesink, Michael Kerres, und Heinz Moser, 33-54. Wiesbaden: VS Verlag für Sozialwissenschaften.

Spanhel, Dieter. 2011. «Medienbildung als Grundbegriff der Medienpädagogik. Begriffliche Grundlagen für eine Theorie der Medienpädagogik.» In Medienbildung und Medienkompetenz, hrsg. v. Heinz Moser, Petra Grell, und Horst Niesyto, 95-120. München: kopaed. doi:10.21240/mpaed/20/2011.09.15.X.

Süss, Daniel, Claudia Lampert und Christine W. Wijnen. 2013. Medienpädagogik. Ein Studienbuch zur Einführung. 2. Aufl. Wiesbaden: VS Verlag für Sozialwissenschaften.

Wieser, Matthias. 2006. "Naturen, Artefakte und Performanzen - Praxistheorie und AkteurNetzwerk-Theorie.» In Verschwindet die Natur? Die Akteur-Netzwerk-Theorie in der umweltsoziologischen Diskussion, hrsg. v. Martin Voss und Birgit Peuker, 95-109. Bielefeld: transcript. 
Wimmer, Michael. 2002. «Pädagogik als Kulturwissenschaft. Programmatische Überlegungen zum Status der Allgemeinen Erziehungswissenschaft.» Zeitschrift für Erziehungswissenschaft, Beiheft 1, Nr. 5: 109-122.

Winter, Rainer. 2004. "Cultural Studies und kritische Pädagogik.» MedienPädagogik. Zeitschrift für Theorie und Praxis der Medienbildung 8: 1-16. http://www.medienpaed.com/ article/view/50

Winter, Rainer. 2011. "Stuart Hall: Die Erfindung der Cultural Studies.» In Kultur. Theorien der Gegenwart, hrsg. v. Stephan Moebius und Dirk Quadflieg, 469-481. Wiesbaden: VS.

Wrana, Daniel, und Antje Langer. 2007. «An den Rändern der Diskurse. Jenseits der Unterscheidung diskursiver und nicht-diskursiver Praktiken.» Forum Qualitative Sozialforschung 8, Nr. 2. http://www.qualitative-research.net/index.php/fqs/article/view/253.

Wrana, Daniel, Marion Ott, Kerstin Jergus, Antje Langer, und Sandra Koch. 2014. «Diskursforschung in der Erziehungswissenschaft.» In Diskursforschung. Ein interdisziplinäres Handbuch, Band 1, hrsg. v. Johannes Angermuller, Martin Nonhoff, Eva Herschinger, Felicitas Macgilchrist, Martin Reisigl, Juliette Wedl, Daniel Wrana, und Alexander Ziem, 224-238. Bielefeld: transcript. 